\title{
Comparative Analysis of Ranking Algorithms Used On Web
}

\author{
Sandeep Suri*, Arushi Gupta and Kapil Sharma \\ Department of Information and Technology, Delhi Technological University Delhi, India \\ sandeepsuri001@gmail.com; arushi2410@gmail.com; kapil@ieee.org \\ *Correspondence: sandeepsuri001@gmail.com
}

Received: 25 $5^{\text {th }}$ January 2020; Accepted: 19 ${ }^{\text {th }}$ February 2020; Published: $1^{\text {st }}$ April 2020

Abstract: With the evolution in technology huge amount of data is being generated, and extracts the necessary data from large volumes of data. This process is significantly complex. Generally the web contains bulk of raw data and the process of converting this data to information mining process can be performed. At whatever point the user places some inquiry on particular web search tool, outcomes are produced with respect to the requests which are dependent on the magnitude of the document created via web information retrieval tools. The results are obtained using calculations and implementation of well written algorithms. Well known web search tools like Google and other varied engines contain their specific manner to compute the page rank, various outcomes are obtained on various web crawlers for a same inquiry because the method for deciding the importance of the sites contrasts among number of algorithm. In this research, an attempt to analyze well-known page ranking calculation on the basis of their quality and shortcomings. This paper places the light on a portion of the extremely mainstream ranking algorithm and attempts to discover a better arrangement that can optimize the time spent on looking through the list of sites.

Keywords: Time Rank; Hybrid Rank; Web Mining; TF-IDF; EigenRumor; TagRank; Weighted Page Rank

\section{Introduction}

Web Pages refer to the information that is stored on the web. While using internet we come across trillions of URLs, many being actively operated. Filtering this gigantic data according to a question/query is rarely a simple process. Thus, proper pre-processing of the data present in the web pages is necessary for retrieving the correct results. All the web pages are interconnected to one another [1]. In order to retrieve the significant data, first the mining must be done, as it is an important step. Figure 1 delineates the KDD process for the web. Web mining process is known as the extraction of information from the raw data on the web. This can be thought as a child set of the data mining. This can be obtained, using 3 main methodologies:
a. Web structure mining
b. Web usage mining
c. Web content mining

Figure 2 delineates the total scientific categorization of web mining. Web structure mining is defined as the process of identifying the structure data from the vast web data. The basic layout of the web reports is of web pages as hub, and hyperlink are the edges associating relevant pages. This essentially gives the synopsis of a specific page [2]. It helps in deciding scores dependent on the

Sandeep Suri, Arushi Gupta and Kapil Sharma "Comparative Analysis of Ranking Algorithms Used On Web", Annals of Emerging Technologies in Computing (AETiC), Print ISSN: 2516-0281, Online ISSN: 2516-029X, pp. 14-25, Vol. 4, No. 2, $1^{\text {st }}$ April 2020, Published by International Association of Educators and Researchers (IAER), DOI: 10.33166/AETiC.2020.02.002, Available: http://aetic.theiaer.org/archive/v4/v4n2/p2.html. 
number of connections that are in-coming and out-passing by considering the significance of the hubs through which the connections are inbound and outbound [2]. Calculation of the ratio of inbound and outbound links of the data is essential for assignment [3]. Web Content Mining furnishes us the coercion of information from web data. It has sound, video, picture, chart, content, list, sets and so on in the form of data. With the progression of innovation, the more information is developing quickly. Over the decade the volumes of data has increased quite significantly [4]. The storage of primary catchphrases, their general recurrence of the keyword and ordering portrays its focal usefulness that is utilized via web crawlers.

Web Usage Mining gains client's comprehension of web use propensities. This implies that which of the websites are effectively being used on frequent interval and which are picking up ubiquity. It comprises tracking down the user's histories, logs or any activity. Artificial Intelligence, big data, social diagram matrices, traffic monitoring, etc. give the best outcome in this domain [5]. It helps in anticipating the forthcoming patterns dependent on present and previous situations. Different logical records have likewise been assessed utilizing the page rank algorithms [6].

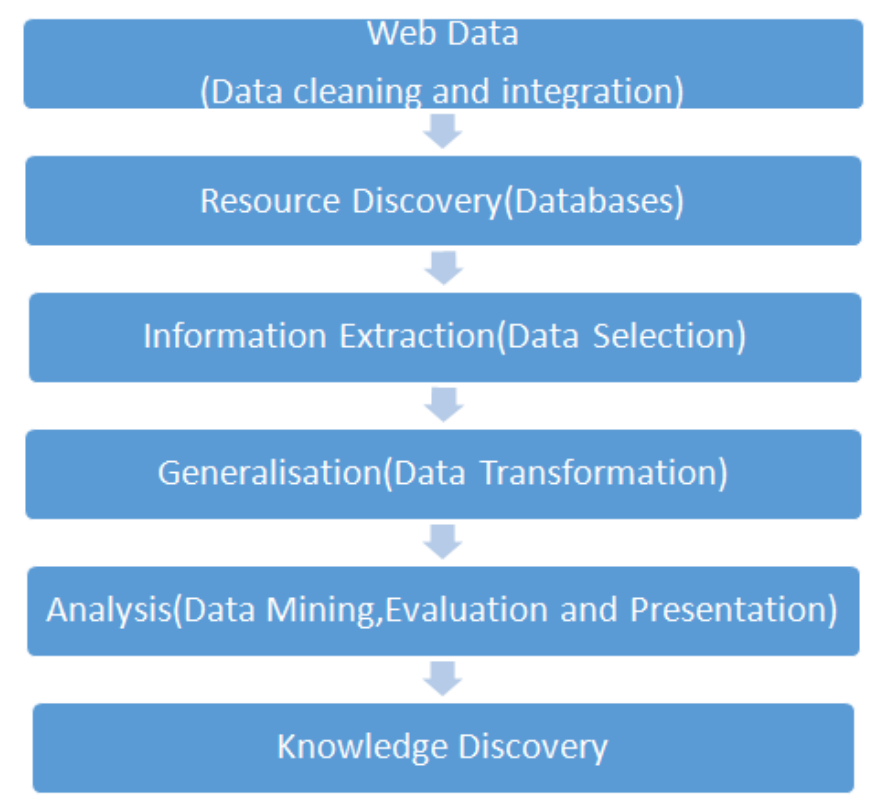

Figure 1. Web Mining Knowledge Discovery Process.

To get the accurate results for the query, endeavours have been devised to get a generalized and optimal algorithm for the ranking. There is a need to comprehend the quick expand in the input provided by user [7]. This research helps a portion of famous ranking algorithms which are already present in the market for utilization and their deficiencies. Different advantages \& restrictions of different pageranking calculations have been studied and worked on in this research contributing the development of a hybrid approach giving better performance rate, optimized time and its effectiveness.

\section{Page Rank Algorithm}

It is a web mining technique given by Google which was originally made for ranking various websites [8]. Larry Page and Sergey Brin are famous computer whizzes who developed the method. It studies the significance of the website pages and in this manner assigns them. As indicated by Google, PageRank [9] is a system that works to look at an approximate of the fundamental sites by checking the quantity and quality of associations with a page [10]. It is accepted that increasingly critical sites will most likely acquire more associations from different sites. This algorithm works with the collaboration of several others to determine the best outcome for the query. Being the focal piece of the world's most mainstream web search tool, it rouses the need to understand the computational information. Selection of web pages shouldn't be restricted to its uniform distribution as it can reduce the convergence rate. 


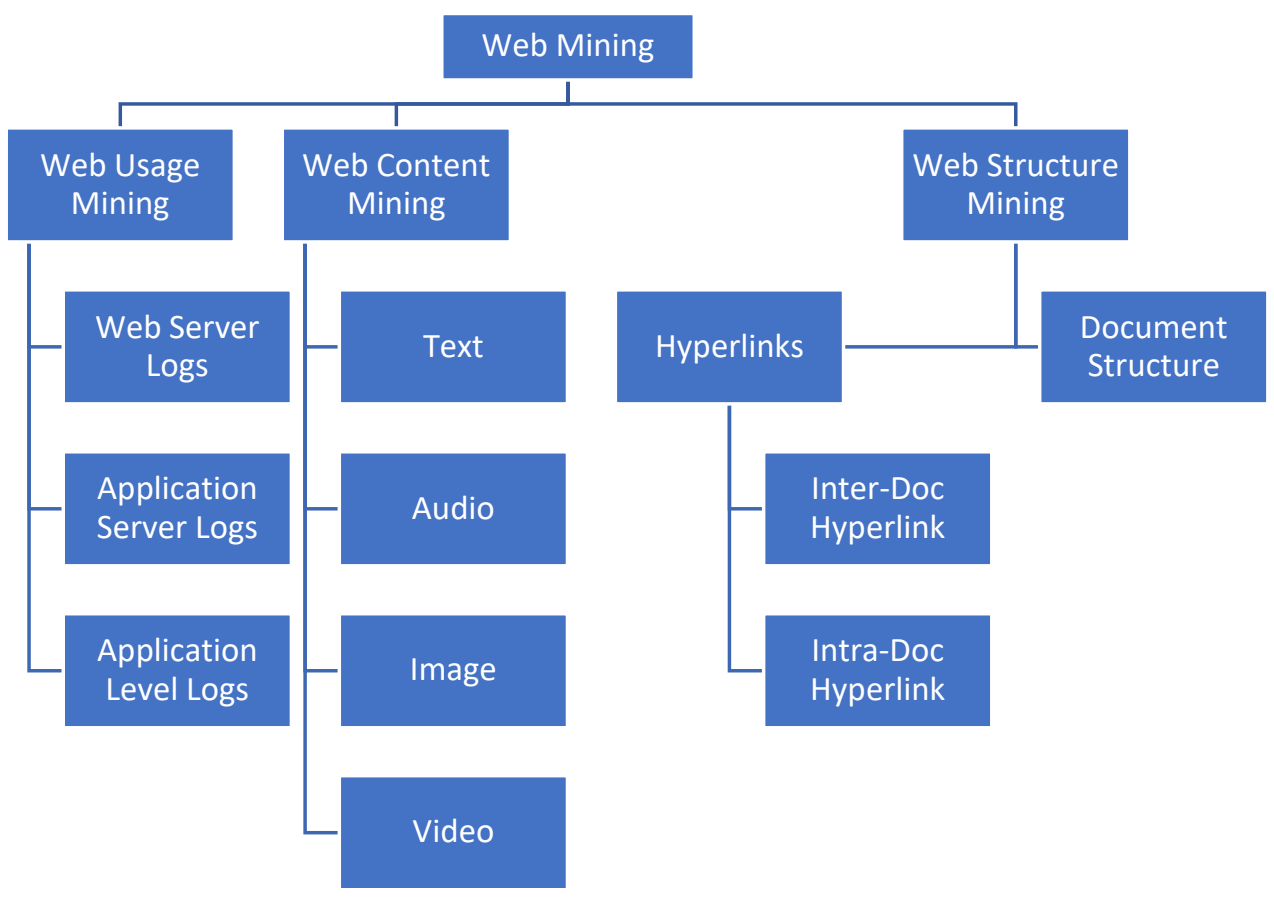

Figure 2. Web Mining Methodologies.

Dividing this calculation into stages helps to comprehend this more effectively. Let $\mathrm{PR}(\mathrm{P})$ be the page rank of page $P$ which can be estimated as the quantity of inbound links towards page $P[6]$. Instinctively, the thought is that in the event coming to page $\mathrm{P}(\mathrm{i})$, at that point presence of a route from $\mathrm{P}(\mathrm{i})$ to $\mathrm{P}$ implies that client is probably going to the page $\mathrm{P}$. If the quantity of links from different pages coordinated towards page $\mathrm{P}$ is high then higher will be the likelihood to get to the $\mathrm{P}$.

$$
P R(P) \propto \sum_{P_{\mathrm{i}} \rightarrow P} \frac{P R(i)}{L(P)}
$$

Let us consider the damping factor where $d$ stands for the ability of user to traverse to any location from $\mathrm{P}(\mathrm{i})$ rather than to $\mathrm{P}$. The formula is,

$$
P R(P)=d \sum_{P_{\mathrm{i}} \rightarrow P} \frac{P R(i)}{L(P)}+\frac{1-\mathrm{d}}{N}
$$

The damping factor (d) is estimated around $0.849, \mathrm{~N}$ is the cumulative amount of website pages, $\mathrm{PR}()$ is the rank of page $\mathrm{P}(\mathrm{i}), \mathrm{L}(\mathrm{)})$ stands for cumulative amount of links outbound from $\mathrm{P}(\mathrm{i})$ and $\mathrm{PR}(\mathrm{P})$ can be defined as the page rank of page $\mathrm{P}[11]$.

Steps of the algorithm is listed below,

1. Initialization $P R(P)=100 \% \frac{1}{N}$

2. Let us consider page $\mathrm{P}$,

$$
P R(P)=d \sum_{P_{\mathrm{i}} \rightarrow P} \frac{P R(i)}{L(P)}+\frac{1-\mathrm{d}}{N}
$$

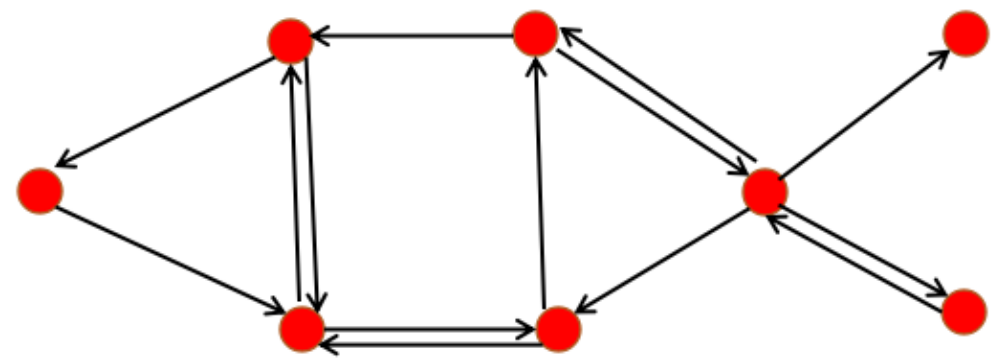

Figure 3. Interconnection of Pages

Page Rank algorithm is a framework given by Google. However in the overhead calculation, they can't work proficiently if isolated [12]. Given an assumption that there exists a trustworthy blog 
with page rank better than the others yet the new substance added to that blog isn't important, so there must be some instrument to go past the connection investigation for this purpose [9].

\section{Weighted Page Rank Algorithm}

Weighted Page Rank is an advanced version of Page Rank given to beat the principle irregularity. W. Xing and A. Ghorbani suggested the given algorithm. On comparing the above two algorithms, in PageRank, positioning of $\mathrm{PR}(\mathrm{P})$ for $\mathrm{P}$ in the web is appropriated similarly among all the outgoing connections yet not all connections are similarly significant and vital[13]. At this point, no motivation is left to share the rank equitably among its outbound connections. This was one of the reasons for the rise of Weighted Page Rank. Distribution of the rank is performed on the basis of the page significance [11]. Ranks are allotted by offering loads to all in and out connections that are meant for Win $(\mathrm{u}, \mathrm{v})$ \& Wout $(\mathrm{u}, \mathrm{v})$ separately. Win $(\mathrm{u}, \mathrm{v})$ is said to be the weight of inbound connection among $\mathrm{u}$ and $\mathrm{v}$ which can be calculated by finding ratio of the total inbound connections of page $\mathrm{v}$ to all in-connections from all directions for page $\mathrm{u}$.

$$
W^{\text {in }}(u, v)=\frac{I_{\mathrm{v}}}{\Sigma_{\mathrm{p}} \in_{\mathrm{r}(u)} I_{\mathrm{p}}}
$$

Where,

Iv stands for the cumulative sum of page $v$ incoming links.

Ip stands for the cumulative sum of page $\mathrm{p}$ incoming links.

Wout $(\mathrm{u}, \mathrm{v})$, stands for the weight of outbound connections among $\mathrm{u}$ and $\mathrm{v}$ which can be calculating the ratio of total quantity of outbound connections of $v$ to the cumulative count of outbound connections of orientations for $\mathrm{u}$ [14].

$$
W^{\text {out }}(u, v)=\frac{O_{\mathrm{v}}}{\Sigma_{\mathrm{p}} \in_{\mathrm{r}(u)} O_{\mathrm{p}}}
$$

After identifying the total significance of all the web pages final equation is as follow:

$$
P R(u) \text { 园 }(1-d)+d \Sigma \operatorname{PR}(\mathrm{v}) W^{\text {in }}(u, v) W^{\text {out }}(u, v)
$$

The fundamental issue with respect to the vitality and significance of connections is settled by Weighted Page Rank yet at the same time, there are a number of inconsistencies like pinpoint ordering, inquiry independence \& page rank estimation [15].

\section{TF-IDF Algorithm}

Term Frequency - Inverse Document Frequency (TF-IDF), a basic position computation that utilizes content mining for determining the main keywords of reports. Web crawlers generally use the subordinates of TF-IDF for finding these catchphrases in websites. Essentially, it is a result of two factual key terms TF and IDF [16]. The number of times a particular term in a given expression is showing up in the content is term recurrence. Whereas, the words like 'the', 'a', 'an', 'of', etc. which are exceptionally regular and lessen the impact of different words, for this IDF is used. IDF factor can be thought of as a normaliser, it diminishes the weight of those words that occur frequently while expands the weight of lesser used identifiers. The IDF report is numerical assessment of information given by the words based on the basis of their occurrence [12].

$T F-I D F(t, d, D)=T F(t, d) * I D F(t, D)$

Where,

$\mathrm{TF}=$ Term recurrence,

The formula for IDF is given by accompanying equation,

$$
I D F=\log \left(\frac{N}{D F(w)}\right)
$$

Subsequent, we get,

$$
T F-I D F(w)=\operatorname{count}(w, d) * \log \left(\frac{N}{D F(w)}\right)
$$

Using important varieties of TF-IDF it helps in ordering the key terms on web crawler's catalogs. In any case, this calculation alone isn't adequate for ranking however it provide some relief in 
building a few functionalities of the page ranking algorithm[17]. In the event that we consider the two calculations exclusively, at that point TF-IDF is better method in correlation of PageRank in the light of the fact that the given algorithm gives keyword centred searching while PageRank is fundamentally centred around scores dependent on interface investigation. Record recurrence is a quantitative investigation process [4].

\section{HITS Algorithm}

Hyperlink Induced Topic Search algorithm is also knows as HITS algorithm, it is characterized in the form of connection examination algorithm which ranks website at runtime query processing. While performing the HITS computation, right off the bat most important pages are retrieved by utilizing content-based computation and is called root set [18]. During HITS calculation the index set is acquired utilizing all connections passing by the root set.

The method for creating root and dependent interconnections of base set is exhibited in figure 4 [10].

Centre point and leader are characterized with respect to each other. Centre point score is the summation of all the central leader scores which is indicated by that centre point, while authority worth can be determined by the summation of absolute centre point esteems that are highlighting that leader [1]. In figure 5, Hubs and leader locales are depicted.

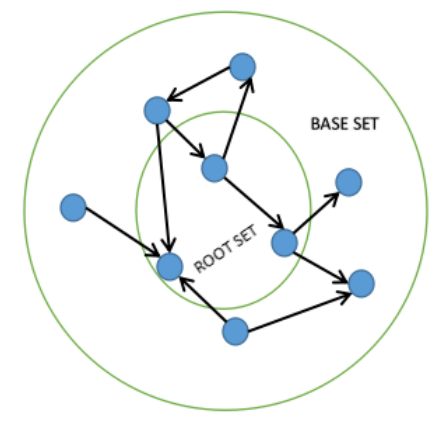

Figure 4. Root Set \& Base Set.

Two most essential strides in Hyperlink Induced Topic Search calculation are validation mechanism of central point and leader score.

- Leader score is refreshed by relegating it to sum of centre point esteems which are getting highlighted. On updation of all $\mathrm{p}$ auth(p),

$\operatorname{auth}(p)=\sum_{i=1}^{n} h u b(i)$

- Hub score is refreshed using the summation of position esteems that are getting pointed at. On updation of all $\mathrm{p}$ hub(p),

$h u b(i)=\sum_{i=1}^{n} \operatorname{auth}(p)$

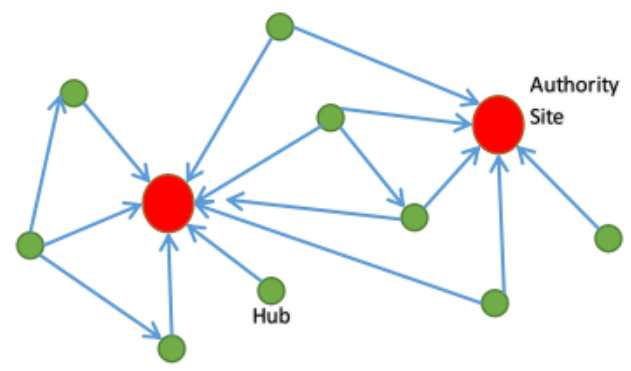

Figure 5. Sites of Hubs and Leader/Authority. 
This functions in the following manner:

- $\quad$ Each centre point and leader/authority is relegated a value equivalent to 1 .

- Updating of the value of centre and leader/authority is done.

- Every central point and authority score is normalized.

- Normalization: Normalization of the considerable number of centre points and leader/authority value is standardized through isolation by the square base of aggregate of all values of centre point and all values of power individually [19].

- Iteration from second step can be performed whenever required.

Hits calculation is well known due to its capacity for ranking site at inquiry time and giving optimal solutions to the clients query [3]. Through this one can consolidate with another data recovery framework to give better results. Though there still exits some drawbacks that are related to this, for example it still has greater reaction time to all the calculation which are done at inquiry time. For some exceptional instances HITS calculation may endure some issues such as theme float (unequivocally associated superfluous pages at root level), immaterial centre points and shared fortification among centres and specialists [8].

While performing the HITS computation, right off the bat most important pages are retrieved by utilizing content-based computation and is called root set [18]. During HITS calculation the index set is acquired utilizing all connections passing by the root set.

The method for creating root and dependent interconnections of base set is exhibited in figure 4 [14].

Centre point and leader are characterized with respect to each other. Centre point score is the summation of all the central leader scores which is indicated by that centre point, while authority worth can be determined by the summation of absolute centre point esteems that are highlighting that leader [1]. In figure 5, Hubs and leader locales are depicted.

\section{HITS Algorithm}

Blog with respect to structure point of view is defined in number of ways; here we are not concerned about content of blog as the content of blog may vary from user to user and from demand to demand. Thus a blog may contain following things and follow [16] this structure in general. A person who manages the blog, updates and writes the blog is also known as blogger.

A. A top page and set of entries that contain blog and content. Usually a blog is maintained by a single blogger and updated by a single blogger. This also depends on nature of blog and content that blog contains. There may be multiple users who can update blog and manage it.

B. There are usually many links from the top of blog to each blog entry that are linked together and they have a permanent Uniform Resource Locator. Each blog is linked to another blog. This linking is used to help user to maintain the flow of content which also provide synchronization to the person who is reading and browsing content on blog.

C. When blog entries are updated, content is added and updated then user may get notification ping. It depends on blogger whether he want to notify his reader and user about the update of blog or not. If there is a need of notifying user and reader it can be done using ping. 
D. A special mechanism is provided so that reader can track-back, go back in a trace and flow which it followed $\mathrm{p}$ while reaching to a particular blog.

Blogging has become a trend in today's growing world and with this arises the challenge for providing good content for the given query. The above discussed algorithms are exceptionally encouraging in ranking an incentive of online journals however some confinements still exit on using these calculations [7] for online journals. The ranking values of blogs are selected by ranking algorithm is regularly low. Therefore ranking the pages based on their significance isn't a good process. In order to determine this adversity, an EigenRumor algorithm [13] is suggested to assign blog rank. This algorithm then ranks each site by calculating the score of the central point and authority of the bloggers contingent upon the computation of eigen-vector.

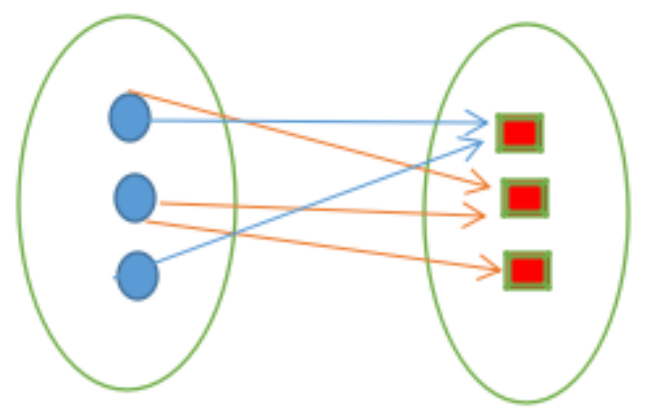

Figure 6. EigenRumor community model.

There are various methods and parameter to rank a blog. Ranking a blog is dependent on various methods and parameters. They are as follows:-

1) Semantics of Ranking

a) Recentness / freshness

b) Strength and support from the community

c) Specific attribute includes e.g., use-fullness, innovative, etc.

2) Space of ranking
a) All blogs
b) Blogs specific provider
c) Blog that are sending a ping or notification on update

3) Source of evaluations collected
a) Hypertext link
b) Hyper-linking, e.g. trace-back
c) Collection of votes
d) Natural language processing and analysis
e) Votes Collections.

4) Ranking a Subject
a) Article that are directly related to blog
b) Articles and post that are indirectly related to blog
c) Article refereed by and refereed to blog
d) Product, Services and Goods material resources refereed to or by -Blog. 


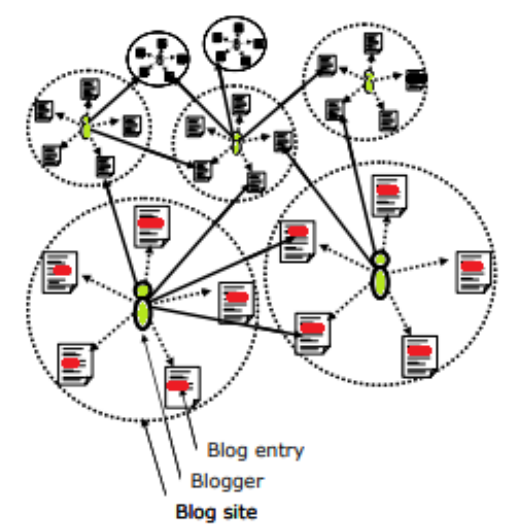

Figure 7. Mapping Blog community.

This methodology likewise empowers to allocate a higher score when the blog section is presented by a blogger who has been acknowledged a ton of consideration before, regardless of whether the passage itself has no in-joins from the outset. This is an attractive component of blog rankings since blog space are viewed as a network where talking about new points. [17]

\section{Query Dependent Ranking Algorithm}

A query is a question, doubt or confusion regarding a particular and specified thing. A query ranking dependent framework can be constructed by constructing a framework for each query and its training query [5]. When a query is filed up by user or person then similar query known as testing query is generated by framework which is very similar to the actual query asked and its measure is being evaluated and rank is being determined. The flowchart of the proposed framework is as follows in figure.

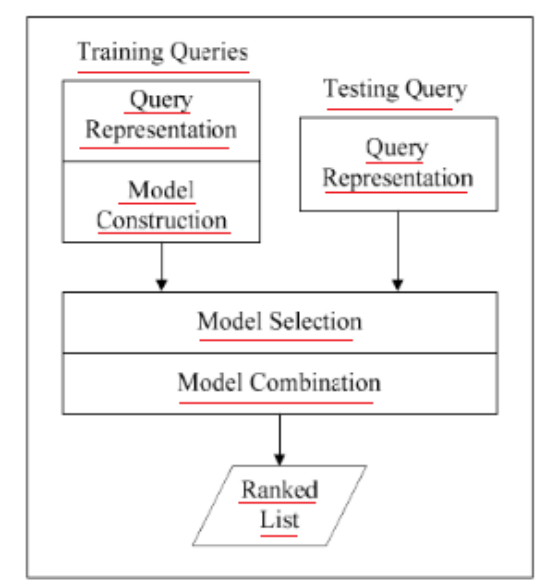

Figure 8. Query Ranking Framework Flowchart.

Using this algorithm we work on query dependent ranking algorithms for providing the outcomes for the query [9]. In this methodology, a straightforward comparability calculation is utilized for gauging measure among various queries. One model can be developed for ranking each training set with the comparing document. [16] Suppose at any point problem arises, then at that point records are extricated and ranking on the basis of the rank scores. In this calculation, it is the mixture of multiple models of comparable preparing inquiries [19]. Exploratory outcomes show that the question subordinate positioning calculation is superior to different calculations. 


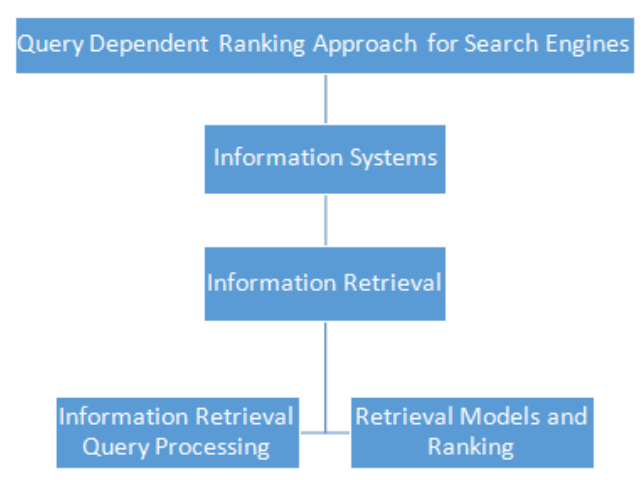

Figure 9. Query dependent ranking process model.

\section{TagRank Algorithm}

TagRank [16] is one of the most unique methods for ranking the website page dependent on social slangs which is proposed by S. Jie, Chen, Z. Hui, Sun Rong-Shuang, Z. Yan and He Kun. This method determines heat of classes by utilizing time factor of recent information tag of the source and activities of the web users. This gives a verification technique far superior than others for ranking the pages [17]. The outcomes of algorithm are accurate and records new data assets. Future work toward the current path is used for the co-occurrence factor of tag which decides the load of the tag and can be improved by utilizing semantic relationships for the co-event classes.

Steps of the calculations are as follows:

- Extract the tags from the websites

- Pre-treatment of the tag is done

- Calculation of number of times the tag is annotated

- Evaluate the initial value of all the tags

- Calculate the tag's heat pi, heat factor and the final heat factor in a time slice.

- Determine the value of TagRank.

Tag Rank algorithm is as follows.

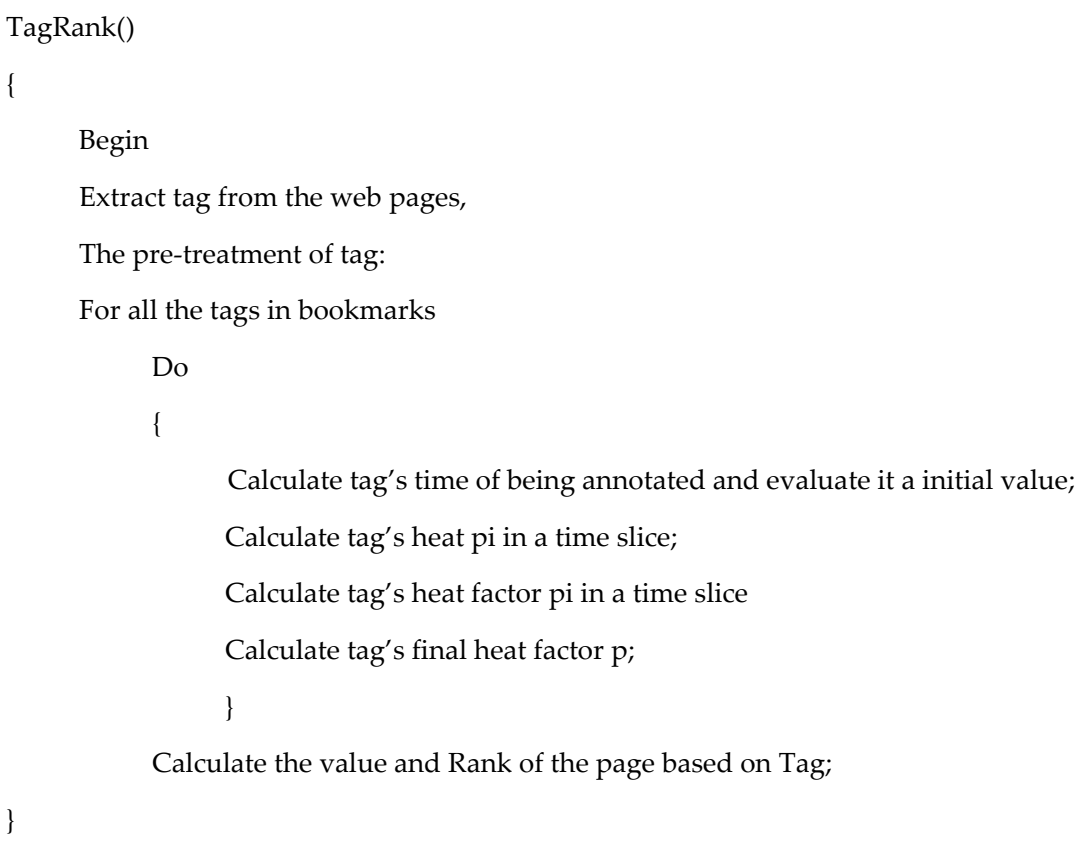




\section{Time Rank Algorithm}

Time-Rank algorithm is used for recuperating the score and value by utilizing the visit time for page [15] Here we have estimated the time to visit the page subsequent to apply unique and recuperated strategies for rank calculation to think about the level of significance to the users. The time factor to build the precision of the website page positioning is used in the algorithm. Because of the system utilized in this calculation, it tends to be thought to be a mix of substance and connection structure. [20] The consequences of this calculation are palatable and in concurrence with the applied hypothesis for building up the calculation.

\section{Comparison Table of Ranking Algorithms}

Table 1. Comparison Table of Various Ranking Algorithms

\begin{tabular}{|c|c|c|c|c|c|}
\hline Algorithm & Page Rank & HITS & $\begin{array}{l}\text { Weighted Page } \\
\text { Rank }\end{array}$ & Eigen Rumor & $\begin{array}{l}\text { Web Page Rank } \\
\text { Using LINK }\end{array}$ \\
\hline Main Technique & $\begin{array}{l}\text { Web Structure } \\
\text { Mining }\end{array}$ & $\begin{array}{l}\text { Web Structure, } \\
\text { Content Mining }\end{array}$ & $\begin{array}{l}\text { Web Structure } \\
\text { Mining }\end{array}$ & $\begin{array}{l}\text { Web Content } \\
\text { Mining }\end{array}$ & $\begin{array}{l}\text { Web Structure, } \\
\text { Content Mining }\end{array}$ \\
\hline Methodology & $\begin{array}{l}\text { Score of Page } \\
\text { at time of } \\
\text { index }\end{array}$ & $\begin{array}{l}\text { Hubs and } \\
\text { Authorities of } \\
\text { relevant Page }\end{array}$ & $\begin{array}{l}\text { Weight of web } \\
\text { page on basis of } \\
\text { input and } \\
\text { outgoing links }\end{array}$ & $\begin{array}{l}\text { Uses } \\
\text { Adjacency } \\
\text { Matrix from } \\
\text { agent }\end{array}$ & $\begin{array}{l}\text { Gives different } \\
\text { weight to web } \\
\text { based on } \\
\text { attributes }\end{array}$ \\
\hline Input & Back Links & Content, Links & Links & Agent Obj & Links \\
\hline Relevancy & Less & More & Less & High & More \\
\hline Result Quality & Medium & Less than PR & Higher than PR & $\begin{array}{l}\text { Higher than } \\
\text { PR and HITS }\end{array}$ & Medium \\
\hline Time & $\mathrm{O}(\log n)$ & $<\mathrm{O}(\mathrm{E}|\mathrm{G}|)$ & $<\mathrm{O}(\log \mathrm{n})$ & $<\mathrm{O}\left(\mathrm{n}^{*} \mathrm{n}\right)$ & $\mathrm{O}(\log n)$ \\
\hline Importance & $\begin{array}{l}\text { High, Back } \\
\text { Link are } \\
\text { considered }\end{array}$ & $\begin{array}{l}\text { Moderate, Hub and } \\
\text { Authority score are } \\
\text { used }\end{array}$ & $\begin{array}{l}\text { High, page are } \\
\text { sorted via } \\
\text { importance }\end{array}$ & $\begin{array}{l}\text { High for blog } \\
\text { ranking }\end{array}$ & Moderate \\
\hline Limitation & $\begin{array}{l}\text { Result at time } \\
\text { of indexing not } \\
\text { at query time }\end{array}$ & $\begin{array}{l}\text { Topic drift and } \\
\text { efficiency problem }\end{array}$ & $\begin{array}{l}\text { Relevancy is } \\
\text { ignored }\end{array}$ & $\begin{array}{l}\text { Usually for } \\
\text { Blog Ranking }\end{array}$ & $\begin{array}{l}\text { Relative position } \\
\text { was not so } \\
\text { effective }\end{array}$ \\
\hline
\end{tabular}

\section{Concluding Discussions}

Based on algorithm and input used the various ranking algorithm shows different results. These result vary on various technique applied and on their methodology as well. Google uses Page rank algorithm in its search engine whereas in blogging Eigen-rumor algorithm is used. Tag algorithm is being widely used to take help from tag and give ranking to algorithm. Hence there is a need of one hybrid algorithm which can do all those work and compute ranking of various thing. Hybrid algorithm should find rank in such a way that it give efficient and optimized result at various parameters and it should also work in worst cases as well.

\section{References}

[1] Suri, Sandeep, Arushi Gupta, and Kapil Sharma. "Comparative Study of Ranking Algorithms." In 2019 International Conference on Computing, Electronics \& Communications Engineering (iCCECE), pp. 73-77, IEEE, 2019, Available: https://ieeexplore.ieee.org/document/8941989.

[2] Nomura, Saeko, Satoshi Oyama, Tetsuo Hayamizu, and Toru Ishida. "Analysis and improvement of hits algorithm for detecting web communities." Systems and Computers in Japan 35, no. 13 (2004): 32-42, Available: https://onlinelibrary.wiley.com/doi/abs/10.1002/scj.10425. 
[3] Kashyap, Satyam, Ashish Tripathi, and Kapil Sharma. "Analysis and Ranking of Software Engineering metrics." In 2015 2nd International Conference on Computing for Sustainable Global Development (INDIACom), pp. 1654-1659, IEEE, 2015.

[4] Ding, Chris HQ, Hongyuan Zha, Xiaofeng He, Parry Husbands, and Horst D. Simon. "Link analysis: hubs and authorities on the World Wide Web." SIAM review 46, no. 2 (2004): 256-268, Available: https://epubs.siam.org/doi/10.1137/S0036144501389218.

[5] Page, Lawrence and Brin, Sergey and Motwani, Rajeev and Winograd, Terry. "The PageRank citation ranking: Bringing order to the web", Stanford InfoLab, January 29, 1998, Available: http://ilpubs.stanford.edu:8090/422/1/1999-66.pdf.

[6] Agrawal, S. K., and Kapil Sharma. "Intelligent Software Defined Atmospheric Effect Processing for 5th Generation (5G) Millimeter Wave (MMWave) Communication System.", International Journal of Wireless and Microwave Technologies(IJWMT), 8(2), 2018, DOI: 10.5815/ijwmt.2018.02.02.

[7] Tripathi, Ashish Kumar, Kapil Sharma, and Manju Bala. "A novel clustering method using enhanced grey wolf optimizer and mapreduce." Big data research 14 (2018): 93-100.

[8] Zheng, Olivier Z., Maaruf Ali, and Kashinath Basu. "Comparing the Complexity of Two Network Architectures." Annals of Emerging Technologies in Computing (AETiC), Print ISSN (2017): 2516-0281, Available: $\underline{\text { https://papers.ssrn.com/sol3/papers.cfm?abstract } \mathrm{id}=3504166 .}$

[9] Lee, Lian-Wang, Jung-Yi Jiang, ChunDer Wu, and Shie-Jue Lee. "A query-dependent ranking approach for search engines." In 2009 Second International Workshop on Computer Science and Engineering, vol. 1, pp. 259-263. IEEE, 2009, Available: https://ieeexplore.ieee.org/document/5403423.

[10] Xing, Wenpu, and Ali Ghorbani. "Weighted pagerank algorithm." In Proceedings. Second Annual Conference on Communication Networks and Services Research, 2004, pp. 305-314. IEEE, 2004, Available https://ieeexplore.ieee.org/document/1344743.

[11] Waheed, Faisal, and Maaruf Ali. "Hardening CISCO Devices Based on Cryptography and Security Protocols - Part One: Background Theory", Annals of Emerging Technologies in Computing (AETiC), Print ISSN: 2516-0281, Online ISSN: 2516-029X, pp. 27-44, Vol. 2, No. 3, 1st July, 2018, DOI: 10.33166/AETiC.2018.03.004, Available: http://aetic.theiaer.org/archive/v2/v2n3/p4.html.

[12] Garg, R. K., Kapil Sharma, C. K. Nagpal, Rakesh Garg, Rajpal Garg, and Rajive Kumar. "Ranking of software engineering metrics by fuzzy-based matrix methodology." Software Testing, Verification and Reliability 23, no. 2 (2013): 149-168.

[13] Fujimura, Ko, Takafumi Inoue, and Masayuki Sugisaki. "The EigenRumor Algorithm for Ranking Blogs " In WWW Workshop on the weblogging ecosystem, 2005, Available:- http://ra.ethz.ch/CDstore/www2005ws/workshop/wf10/fujimura.pdf.

[14] Fu, Hwai-Hui, Dennis KJ Lin, and Hsien-Tang Tsai. "Damping factor in Google page ranking." Applied Stochastic Models in Business and Industry 22, no. 5-6 (2006): 431-444, Available: https://doi.org/10.1002/asmb.656.

[15] Memon, Mashooque Ahmed, Safeeullah Soomro, Awais Khan Jumani, and Muneer Ahmed Kartio. “Big Data Analytics and Its Applications", Annals of Emerging Technologies in Computing (AETiC), Print ISSN: 2516-0281, Online ISSN: 2516-029X, pp. 45-54, Vol. 1, No. 1, 1st July, 2017, DOI: 10.33166/AETiC.2017.01.006, Available: http://aetic.theiaer.org/archive/v1/v1n1/p6.html.

[16] Jiang, Hua, Yong-Xing Ge, Dan Zuo, and Bing Han. "TIMERANK: A method of improving ranking scores by visited time." In 2008 International Conference on Machine Learning and Cybernetics, vol. 3, pp. 16541657. IEEE, 2008. 
[17] Jie, Shen, Chen Chen, Zhang Hui, Sun Rong-Shuang, Zhu Yan, and He Kun. "Tagrank: a new rank algorithm for webpage based on social web" In 2008 International Conference on Computer Science and Information Technology, pp. 254-258. IEEE, 2008, Available: https://ieeexplore.ieee.org/document/4624871.

[18] Suzuki, Atsushi, and Hideaki Ishii. "Distributed randomized algorithms for PageRank based on a novel interpretation." In 2018 Annual American Control Conference (ACC), pp. 472-477. IEEE, 2018, Available: https://ieeexplore.ieee.org/document/8430939.

[19] Xu, Yangbing, Shuai Zhang, Wenyu Zhang, Shuiqing Yang, and Yue Shen. "Research Front Detection and Topic Evolution Based on Topological Structure and the PageRank Algorithm." Symmetry 11, no. 3 (2019): 310, Available: https://doi.org/10.3390/sym11030310.

[20] Walia, Gurjit Singh, Himanshu Ahuja, Ashish Kumar, Nipun Bansal, and Kapil Sharma. "Unified GraphBased Multicue Feature Fusion for Robust Visual Tracking." IEEE transactions on cybernetics (2019), Available: $\underline{\text { https://ieeexplore.ieee.org/document/8745503. }}$.

(C) 2020 by the author(s). Published by Annals of Emerging Technologies in Computing (AETiC), under the terms and conditions of the Creative Commons Attribution (CC BY) license which can be accessed at http://creativecommons.org/licenses/by/4.0. 